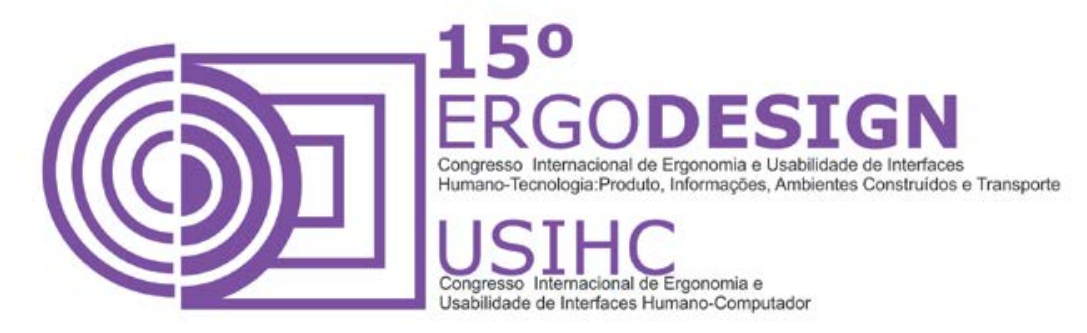

\title{
A INCLUSÃO DO USUÁRIO NO DESIGN DE SISTEMAS DE WAYFINDING: MÉTODOS E TÉCNICAS DE COLETA DE DADOS COGNITIVOS ESPACIAIS
}

\section{USER INCLUSION IN WAYFINDING SYSTEMS DESIGN: METHODS AND COLLECTION TECHNIQUES OF SPATIAL COGNITIVE DATA}

\author{
SMYTHE, Kelli Cristine Assis Silva (1); \\ SPINILLO, Carla Galvão (2) \\ (1) Universidade Federal do Paraná, Ma. \\ e-mail:kellicas@gmail.com \\ (2) Universidade Federal do Paraná, Dra. \\ e-mail:cgspin@gmail.com
}

\begin{abstract}
RESUMO
Este artigo apresenta uma seleção de métodos e técnicas para coleta de dados cognitivos espaciais dos usuários, passíveis de aplicação em sistemas de wayfinding. Para tanto, foi realizada uma revisão de literatura sobre cognição na orientação espacial. Posteriormente, foram levantados métodos e técnicas com abordagem centrada no usuário, os quais foram analisados a partir de suas descrições e, selecionados a partir de palavras-chave e análise interpretativa. Obteve-se uma síntese dos métodos e técnicas com potencial para coleta de dados visando melhorar a eficiência desses sistemas.
\end{abstract}

Palavras-chave: Coleta de Dados; Wayfinding; Cognição Espacial.

\begin{abstract}
This article presents a selection of methods and techniques for data collection on users spatial cognitition, that can be applied in wayfinding systems. To this end, a literature review on cognition was held in spatial orientation. Subsequently, methods andtechniques with user-centric approach were raised, and further analyzed based on their descriptions, and selected from keywords and interpretive analysis. A summary of the methods-techniques with potential for data collection was obtained in order to improve the efficiency of those systems.
\end{abstract}

Keywords: Data Collection; Wayfinding; Spatial Cognition. 


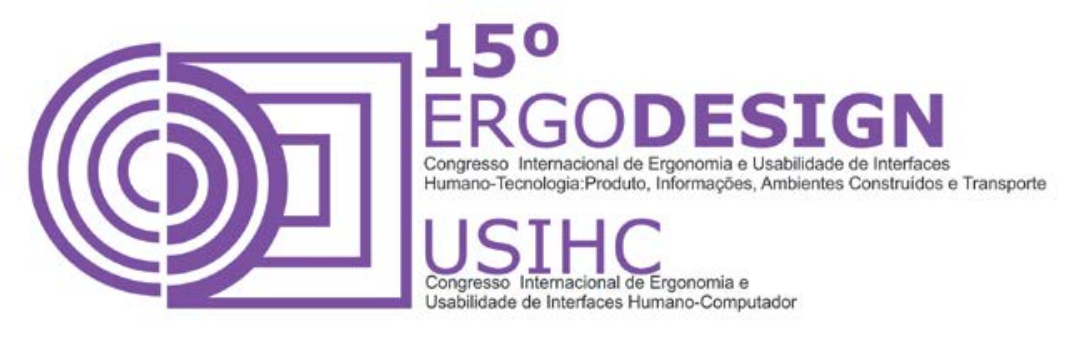

\section{INTRODUÇÃO}

Este artigo é parte integrante da dissertação de mestrado da primeira autora sobre as formas de participação do usuário nos processos de design para sistemas de wayfinding. Orientação espacial ou wayfinding é um processo inerente aos seres humanos já que, para compreendermos e nos deslocarmos no espaço, de acordo com Arthur e Passini (2002), é necessário saber onde estamos, qual o caminho seguir para chegar ao local desejado e então retornar. Os autores acrescentam que, no alcance de um destino, são envolvidos processos perceptuais, cognitivos e comportamentais do usuário (ARTHUR e PASSINI, 2002). A orientação espacial pode ser materializada na utilização de artefatos gráficos - informacionais (como mapas, placas, folders, totens etc.) os quais podem constituir um sistema de wayfinding. De acordo Mollerup (2009), sistemas de wayfinding deficientes podem levar a dificuldades de localização dos usuários nos ambientes, resultando em maior esforço cognitivo e podendo levar ao insucesso na tarefa de localização. Em ambientes complexos como hospitais, tais dificuldades de localização e orientação acentuam-se devido a fatores como: (a) a dificuldade que os usuários encontram em entender a sinalização, neste caso devido a falhas projetuais, questões ergonômicas, representações gráficas e textuais entre outras; (b) o abalo psicológico e estresse a que estão submetidos os usuários em virtude de suas patologias ou pelo motivo que os levaram a tal ambiente (consulta, acompanhamento, exames etc.). Esses fatores acabam prejudicando ainda mais a orientação espacial, uma vez que alteram a percepção e a cognição dos usuários, tornando a busca por um local específico ainda mais angustiante.

No levantamento realizado por esta pesquisa, identificou-se que no design gráfico há escassez de referências sobre como se dá a interação do usuário com o ambiente hospitalar em sistemas de wayfinding. Percebeu-se também que não se têm abordado, na literatura dessa área, métodos e técnicas específicos de coleta de dados implícitos aos projetos de sistemas de wayfinding. Nesse contexto, este trabalho objetivou identificar, a partir da literatura, meios de coletar dados dos usuários para entender como se dá a cognição espacial em um ambiente construído complexo, tendo como foco o ambiente hospitalar. Para tanto, foram levantados, métodos e técnicas capazes de coletar dados cognitivos do usuário durante o processo de orientação espacial em ambientes hospitalares. Tal levantamento incluiu a literatura de diversas áreas como os Estudos sobre os Usuários, Interação Humano-Computador (IHC) e Ergonomia, visto que essas descrevem métodos e técnicas de coleta de dados que têm como foco principal o usuário final. Espera-se que o resultado contribua com profissionais de desenvolvimento e implementação de sinalização (estudantes e profissionais de design/arquitetura) para que obtenham subsídios eficientes que os auxiliem no desenvolvimento projetos de wayfinding.

\section{WAYFINDING E A COGNIÇÃO ESPACIAL}

Diante das dificuldades de orientação e deslocamento dos usuários de ambientes construídos verificou-se necessidade de entender o seu processo cognitivo para identificar quais estratégias de busca ele utiliza. O processo cognitivo na orientação espacial tem sido discutido sob a ótica 


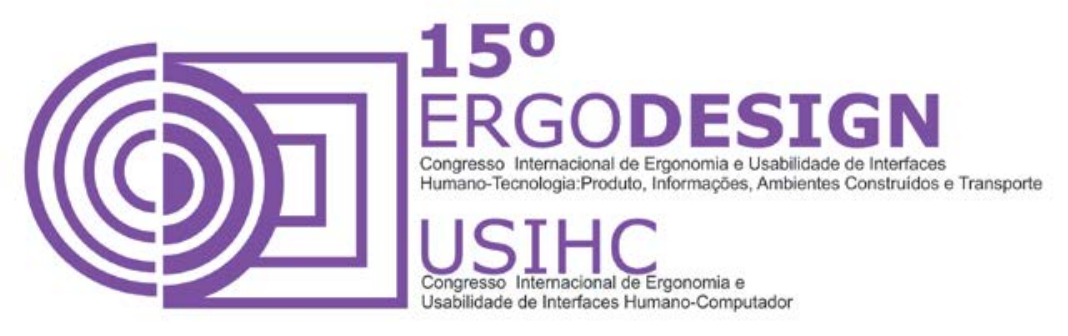

de várias áreas do conhecimento. Segundo os antropólogos Istomin e Dwyer (2009), foi na psicologia que surgiram as teorias sobre orientação humana, com Tolman em 1948, e posteriormente sofreram influências dos estudos em áreas como Arquitetura e Geografia (Lynch, 1999 [1960]; Downs e Stea, 1977) referindo-se à orientação constituinte do processo de wayfinding. As diferentes áreas por vezes se sobrepõem e por vezes se complementam, sendo que a maioria das abordagens considera a existência de mapas cognitivos. Os mapas cognitivos, também chamados de mapas mentais, influenciam a orientação espacial na medida em que se referem ao modelo de representação mental de relações euclidianas que existem entre objetos, de acordo com Istomim e Dwyer (2009). Para o geógrafo Golledge (1999), o mapa cognitivo é um produto, a soma total de informações ambientais armazenados na memória. No Design e na Ergonomia Cognitiva Padovani e Moura (2008), descrevem o mapa coginitivo como uma representação mental da imaginação do usuário juntamente com a sua organização espacial das relações dos elementos (como rotas, distâncias etc.) presentes no ambiente espacial em questão. As autoras acrescentam que essa representação pode ser construída através da interação direta com o ambiente ou da consulta de representações externas (PADOVANI e MOURA, 2008).

Entende-se assim que os mapas cognitivos integram o wayfinding já que este ocorre, de acordo com Arthur e Passini (2002), em três etapas inter-relacionadas: (1) tomada de decisão (plano de ação); (2) execução da decisão (plano em ação) e; (3) processamento da informação (percepção e cognição do ambiente onde se formou a base dos processos anteriores). De modo complementar, Istomim e Dwyer (2009) descrevem que o wayfinding consiste em traçar uma rota em relação aos objetos e lugares codificados em um mapa mental. Ou seja, concluem os autores, durante o curso da viagem de uma pessoa, o movimento é feito de um marco para o outro e a direção do movimento é feita com referência a um mapa mental (ISTOMIM e DWYER, 2009). Segundo Raubal et al. (1997), para que ocorra o processo de orientação espacial ou wayfinding de forma bem sucedida, as pessoas precisam ter várias habilidades cognitivas (como o reconhecimento de objetos) e também conhecimento espacial. Em relação a isso, os psicólogos Siegel e White (1975) afirmam que o conhecimento espacial é elaborado nos níveis de ação no espaço, percepção do espaço e concepção sobre o espaço, todos apresentados de forma integrada. Os autores acrescentam que a construção da representação espacial é realizada a partir da aprendizagem ou conhecimento de: (a) marcos referenciais ou landmarks (como um evento perceptual, ligado às particularidades de cada local, como um reconhecimento do contexto); (b) de rotas (como evento sensório-motor, relativo a caminhos específicos para deslocamento de um ponto a outro, como uma associação de mudanças a partir dos marcos); e; (c) de configuração, também chamado de levantamento (informação métrica sobre a localização relativa e distância estimada entre os marcos referenciais, como a interligação das rotas em rede como um conjunto)(SIEGEL e WHITE, 1975).

Observa-se pelo exposto até aqui, que a convergência das abordagens sobre o processo de orientação/wayfinding ocorre com base nos mapas cognitivos. Pela breve descrição percebe-se a complexidade envolvida no entendimento da cognição na realização do wayfinding. A compreensão do ambiente (orientação) para a ação de deslocamento em si (navegação) contam com alguns auxílios como: indicadores de localização, de circulação, sinalização de 


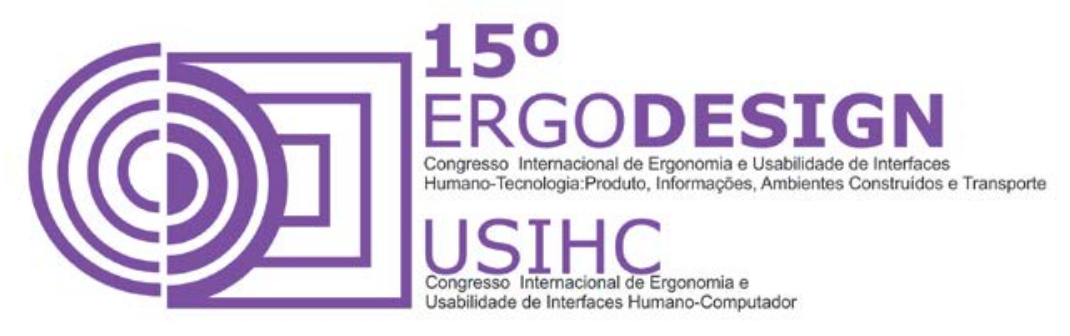

emergência, arquitetura, pontos de referência etc. (PADOVANI e MOURA, 2008). Alguns desses auxílios podem ser materializados em artefatos visuais. Esses compõem os sistemas de wayfinding, a partir da apresentação das informações necessárias à orientação espacial, como placas de sinalização, totens, folhetos e marcos referenciais (elementos arquitetônicos, ambientais naturais ou artificiais). Diante da diversidade de artefatos gráficos possíveis em sistemas de wayfinding, vários estudos têm abordado algumas especificidades, como legibilidade, estudo cromático, formas de representação dos símbolos, posicionamento da sinalização, mapas de rota e você está aqui, placas e totens (BERGER, 2009; GIBSON, 2009; MIJKSENAAR, 1999; O’NEILL, 1999; DEWAR, 1999; ANSI Z535.1, 2006). No entanto, conforme já mencionado, estudos que contemplassem como entender a cognição dos usuários no processo de wayfinding não foram encontrados. Além disso, a ênfase frequentemente dada para problemas de wayfinding, tem se manifestado através de soluções limitadas, segundo Miller e Lewis (2000), com o desenvolvimento de uma sinalização atrativa, sem considerar os muitos fatores que afetam a maneira como as pessoas encontram suas rotas num espaço.

No design, o usuário tem ocupado um novo lugar dentro do processo de desenvolvimento de produtos bi/tridimensionais e serviços quando se adota a abordagem de Design Centrado no Usuário - DCU. Acredita-se que tal abordagem possa trazer eficiência aos projetos de wayfinding já que o DCU caracteriza-se por cinco princípios básicos (ISO 9241-210/2010): (1) Compreensão clara das exigências do usuário, da tarefa e do contexto; (2) Participação ativa dos usuários em toda a concepção e desenvolvimento; (3) Avaliação sob a perspectiva do usuário durante a condução e refinamento; (4) Experiência do usuário e alocação adequada de função entre o usuário e o sistema; (5) Iteração de soluções de design. Com isso torna-se possível "ampliar os requisitos de projeto de modo a incluir as necessidades de usuários, tarefas e contextos de uso do produto em desenvolvimento" (PADOVANI et al., 2012).

Com a identificação da necessidade do entendimento da cognição espacial dos usuários de sistemas de wayfinding e da abordagem do DCU como possibilidade de aumentar esse entendimento (usuário-tarefa-contexto) procurou-se localizar, na literatura, subsídios que possibilitassem aos profissionais de wayfinding conhecer e aplicar essas informações em seus projetos.

\section{ABORDAGEM METODOLÓGICA}

Para realização deste trabalho foi realizado um estudo teórico, a partir de uma pesquisa (levantamento) bibliográfica, de forma descritiva, com o objetivo de identificar quais métodos e técnicas, existentes na literatura, seriam passíveis de aplicação em sistemas de wayfinding. Tal levantamento teve como unidade de análise a cognição espacial, visando entender como esta ocorre nos usuários no ambiente construído. Os dados foram coletados de forma tipológica, selecionados a partir de critérios qualitativos referentes a: (a) serem passíveis de aplicação em sistemas de wayfinding para ambientes construídos, como os hospitais e; (b) serem oriundos da revisão de literatura em Design Centrado no usuário e estudos sobre o usuário (i.e. Design, Arquitetura, IHC, Ergonomia, Geografia). A análise foi realizada de modo interpretativo (GIL, 2010) a partir da descrição dos métodos e técnicas disponibilizados na literatura. 


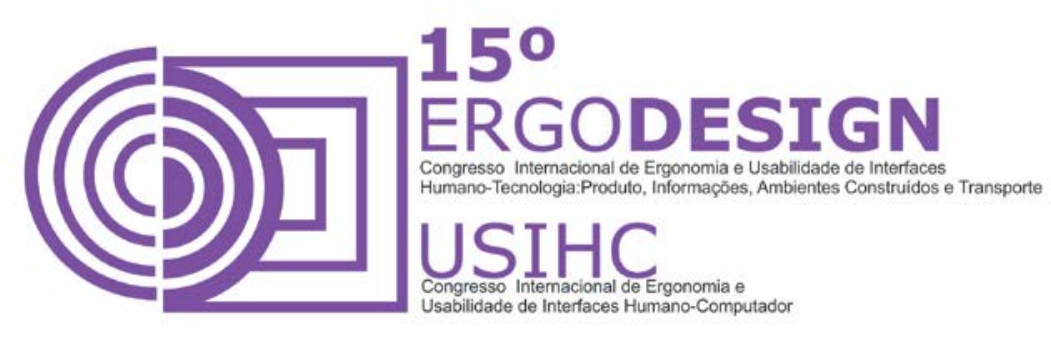

\subsection{Levantamento dos Métodos e Técnicas de Coleta de Dados}

$\mathrm{Na}$ definição do corpus dos métodos e técnicas analisados foram empregadas palavras-chave para a busca, a partir de fontes bibliográficas, em português e inglês, a fim de identificar publicações que tratassem do tema do sentido mais amplo para o mais restrito: processos de design para wayfinding $>>$ wayfinding em ambiente hospitalar $>>$ usuário no processo de design > coleta de dados em processo de design >> coleta de dados dos usuários no design centrado no usuário >> estudo dos usuários.

A busca se deu com a seleção de referências em publicações: (a) livros em mecanismos de busca na internet como o Google Acadêmico; (b) livros impressos; (c) artigos publicados em congressos; (d) dissertações e teses em bases de dados acadêmicas como o Science Direct e o Portal de Periódicos da Coordenação de Aperfeiçoamento de Pessoal de Nível Superior (CAPES). Inicialmente foram selecionados 123 métodos e técnicas os quais foram posteriormente agrupados por similaridade (na terminologia e descrição), obtendo-se 61 métodos e técnicas. Estes podem, segundo análise das descrições de seus autores pelas pesquisadoras, serem utilizados em processos de design de wayfinding com abordagem centrada no usuário. No quadro 1 é representada a síntese com os 61 métodos e técnicas selecionados e agrupados pelas autoras.

Quadro 1 - Listagem dos Métodos e técnicas levantados na literatura já agrupados. 


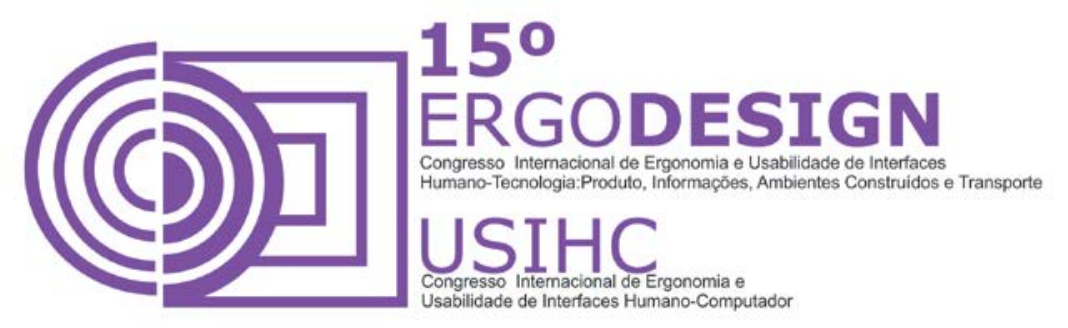

\begin{tabular}{l}
\hline Allocation of function \\
\hline Análise Contextual / Context of use analysis / \\
Contextmapping / Pesquisa contextual \\
(contextual inquiry) \\
\hline análise da tarefa / Task analysis / \\
Cognitive task analysis \\
\hline Análise do incidente crítico / Critical \\
incidents / Técnica do incidente crítico \\
\hline Aval. de co-descoberta \\
\hline Avaliação AUA \\
\hline Avaliação Cooperativa \\
\hline Avaliação heurística / Participativa \\
\hline Braindrawing \\
\hline Brainstorming \\
\hline Brainwriting \\
\hline Buttons Project \\
\hline Caixa Morfológica \\
\hline CARD \\
\hline Cardsorting \\
\hline Casos de uso \\
\hline Cenários / Written Scenario / Scenarios of use \\
\hline Cognitive walkthrough/percurso cognitivo \\
\hline Constelação de Atributos \\
\hline Diagrama de Afinidades \\
\hline Diary keeping / Day in the Life \\
\hline
\end{tabular}

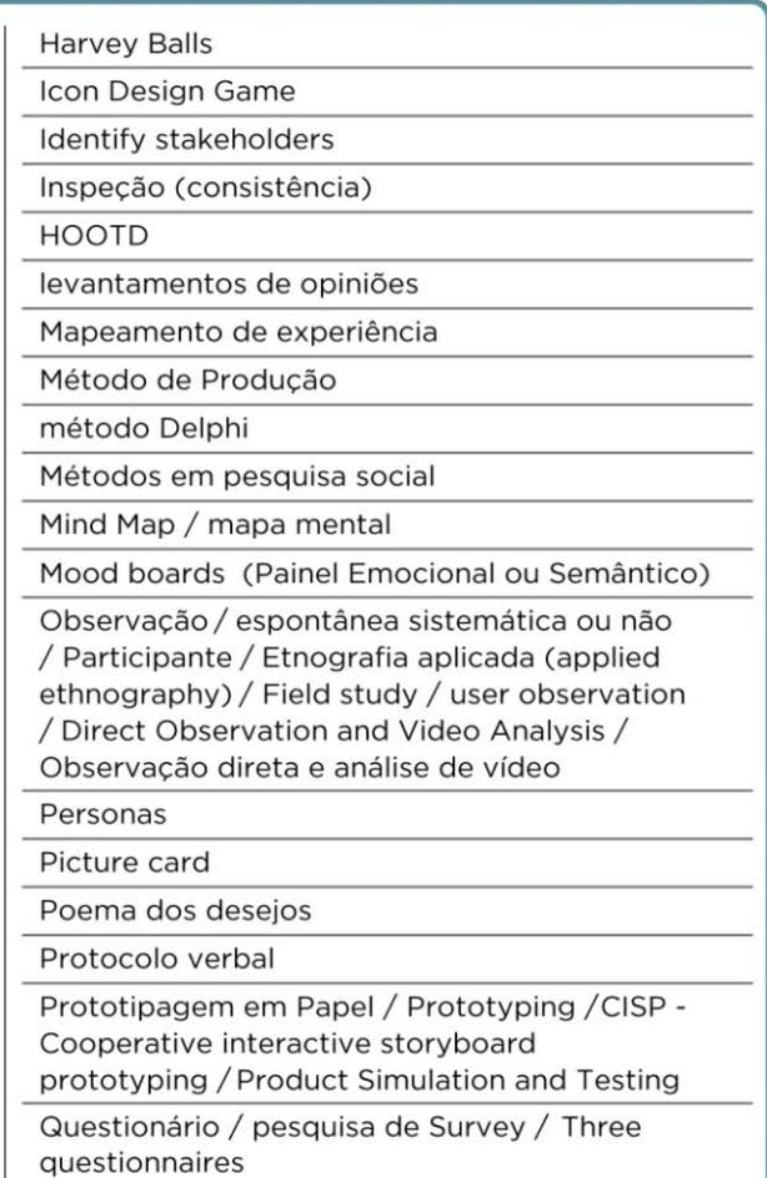

\begin{tabular}{|l}
\hline Diferencial Semântico \\
\hline Entrevista / Entrevista episódica e narrativa / \\
User requirements interview \\
\hline Esboço Cartográfico \\
\hline Essays and drawings analysis \\
\hline Estimativa de Compreensibilidade \\
\hline Estruturação do espaço - Esquemas de imagem \\
\hline Existing system / competitor analysis \\
\hline Eyetracking \\
\hline ETHICS \\
\hline Fly on the Wall \\
\hline Focus group
\end{tabular}

\begin{tabular}{l}
\hline Sense-making \\
\hline Sondas Culturais (Cultural Probes) \\
\hline Task / function mapping \\
\hline Teste de Stress de Navegação ( Stress \\
Navegation Test) \\
\hline Teste de Usabilidade / User, usability and \\
organizational requirements \\
\hline The bridge \\
\hline User cost-benefit analysis \\
\hline Workshops / Storytelling / Priority Workshop \\
\hline
\end{tabular}




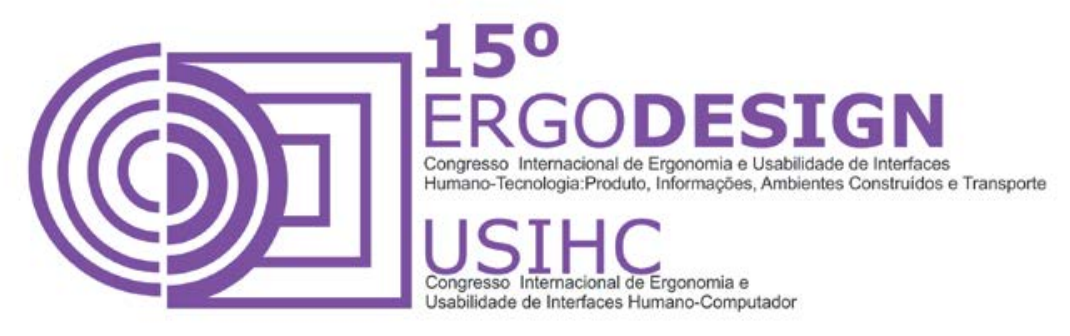

Foram considerados apenas os métodos e técnicas descritos como utilizáveis em estudos dos usuários, design centrado no usuário e em sistemas de wayfinding. Além disso, também foi analisada a norma ISO/TR 16982 (2002) > ISO WD 9241-230: (2009), a qual disponibiliza técnicas de coleta de dados com o foco no usuário. Ao todo foram selecionados 18 autores (mais a ISO) para extração dos métodos e técnicas de coleta de dados dos usuários. O quadro 2 apresenta a listagem dos autores, as publicações utilizadas e os conteúdos abordados pelos mesmos.

Quadro 2 - Listagem dos autores e publicações selecionadas com respectivos conteúdos

\begin{tabular}{|c|c|c|}
\hline № & Autor / Publicação & Conteúdo \\
\hline 1 & $\begin{array}{l}\text { Ardito et al., } 2007 \text { - artigo no manifesto: } \\
\text { Towards the evaluation of UX. }\end{array}$ & $\begin{array}{l}\text { métodos e técnicas para captar a experiência do } \\
\text { usuário no contexto de aprendizagem, ex. ques- } \\
\text { tionário triplo. }\end{array}$ \\
\hline 2 & $\begin{array}{l}\text { Boeijen e Daalhuizen, } 2010 \text { - guia Delft } \\
\text { Design. }\end{array}$ & $\begin{array}{l}\text { diferentes formas de abordar o design (métodos e } \\
\text { técnicas). }\end{array}$ \\
\hline 3 & $\begin{array}{l}\text { Dias e Pires, } 2004 \text { - livro: Usos e usuários } \\
\text { da informação. }\end{array}$ & $\begin{array}{l}\text { estudo sobre os usuários realizado na área da } \\
\text { Ciência da Informação. }\end{array}$ \\
\hline 4 & $\begin{array}{l}\text { Liz Sanders, } 2008 \text { - From User-Centered } \\
\text { to Participatory Design Approaches. }\end{array}$ & $\begin{array}{l}\text { abordagens de design centrado no usuário/partici- } \\
\text { pativo - métodos de coleta de dados de DCU, } \\
\text { design crítico, design e emoção, e design partici- } \\
\text { pativo. }\end{array}$ \\
\hline 5 & $\begin{array}{l}\text { Ferreira, 1997- Design de biblioteca virtual } \\
\text { centrado no Usuário: a abordagem do } \\
\text { Sense-Making para estudos de necessi- } \\
\text { dades e procedimentos de busca } \\
\text { e uso da informação. }\end{array}$ & $\begin{array}{l}\text { descreve o uso do sense-making para compreen- } \\
\text { são de como os usuários buscam a informação. }\end{array}$ \\
\hline 6 & $\begin{array}{l}\text { Fonseca e Rheingantz, } 2009 \text { - artigo: O } \\
\text { ambiente está adequado? Prosseguindo } \\
\text { com a discussão. }\end{array}$ & $\begin{array}{l}\text { técnicas e ferramentas dentro de uma metodolo- } \\
\text { gia que foca nos aspectos físicos, funcionais, } \\
\text { cognitivos e culturais das demandas dos usuários } \\
\text { do ambiente construido. Destaque para o poema } \\
\text { dos desejos. }\end{array}$ \\
\hline
\end{tabular}




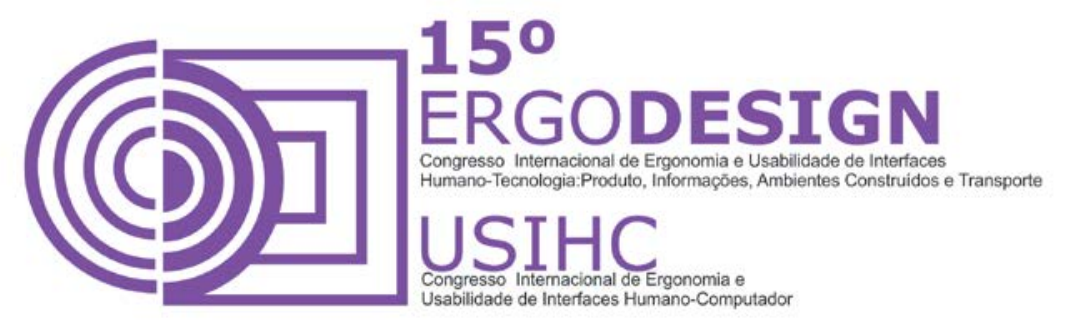

\begin{tabular}{|c|c|c|}
\hline 7 & $\begin{array}{l}\text { Gomes, A. S. et al., } 2008 \text { - artigo: Design } \\
\text { da Interação de Novos Produtos para } \\
\text { TVD: Abordagens Qualitativas. }\end{array}$ & $\begin{array}{l}\text { técnicas de coleta de dados com abordagens } \\
\text { qualitativas de interação. }\end{array}$ \\
\hline 8 & $\begin{array}{l}\text { Gonçalves, } 2012 \text { - artigo: Abordagem } \\
\text { sense-making na ciência da informação: } \\
\text { uma breve contextualização. }\end{array}$ & $\begin{array}{l}\text { métodos de extrair as necessidades informacionais } \\
\text { dos usuários para resolução de problemas. }\end{array}$ \\
\hline 9 & $\begin{array}{l}\text { Guidalli, } 2012 \text { - dissertação: Diretrizes } \\
\text { para o projeto de salas de aula em } \\
\text { universidades visando o bem-estar } \\
\text { do usuário. }\end{array}$ & $\begin{array}{l}\text { técnicas e ferramentas que visam coletar infor- } \\
\text { mações sobre o bem-estar do usuário em um } \\
\text { ambiente construído, entre elas a ferramenta } \\
\text { "constelação de atributos". }\end{array}$ \\
\hline 10 & $\begin{array}{l}\text { ISO/TR 16982, } 2002 \text { > ISO WD 9241-230: } \\
2009 \text { - Norma - User requirements for } \\
\text { ISO Standard: Human-centred design } \\
\text { and evaluation methods. }\end{array}$ & $\begin{array}{l}\text { métodos de usabilidade para aplicação em Design } \\
\text { Centrado no Ser Humano. }\end{array}$ \\
\hline 11 & $\begin{array}{l}\text { Leal, } 2008 \text { - tese: Contribuições da } \\
\text { análise da atividade e da entrevista de } \\
\text { autoconfrontação para os estudos de } \\
\text { usuários. }\end{array}$ & técnicas de estudos sobre os usuários. \\
\hline 12 & $\begin{array}{l}\text { Maguire, } 2001 \text { - artigo: Methods to } \\
\text { support human-centred design. }\end{array}$ & $\begin{array}{l}\text { compilação dos métodos que apóiam o design } \\
\text { centrado no usuário, considerando os sistemas } \\
\text { digitais. }\end{array}$ \\
\hline 13 & $\begin{array}{l}\text { Padovani et al., } 2012 \text { - artigo: Trilhando } \\
\text { o caminho de volta: um estudo da } \\
\text { adaptação de métodos de design } \\
\text { centrado no Usuário para sistemas de } \\
\text { informação digitais visando aplicação } \\
\text { à mídia impressa. }\end{array}$ & $\begin{array}{l}\text { identifica quais métodos e técnicas de DCU } \\
\text { utilizados em sistemas digitais e que podem ser } \\
\text { utilizados em sistemas impressos. }\end{array}$ \\
\hline 14 & $\begin{array}{l}\text { Raubaul et al., } 1995 \text { - artigo: Structuring } \\
\text { Space with Image Schemata: Wayfinding } \\
\text { in Airports as a Case Study. }\end{array}$ & $\begin{array}{l}\text { método de estruturação para compreensão } \\
\text { do espaço em sistemas de wayfinding, a partir } \\
\text { de esquemas de imagem. }\end{array}$ \\
\hline 15 & $\begin{array}{l}\text { Reis, Decanini e Pugliesi, } 2010 \text { - artigo: } \\
\text { Método de esboço cartográfico para } \\
\text { seleção de informação de guia de rota: } \\
\text { um estudo preliminar. }\end{array}$ & $\begin{array}{l}\text { método do "Esboço Cartográfico" para coleta de } \\
\text { informações sobre rotas. }\end{array}$ \\
\hline 16 & $\begin{array}{l}\text { Guilherme Santa Rosa e Ana Maria de } \\
\text { Moraes, } 2012 \text { - livro: Design Participativo. }\end{array}$ & $\begin{array}{l}\text { mais de } 30 \text { métodos e técnicas de coletas de } \\
\text { dados para realização de design participativo. }\end{array}$ \\
\hline 17 & $\begin{array}{l}\text { Baptista e Cunha, 2007- artigo: Estudos } \\
\text { de Usuários: visão global dos métodos } \\
\text { de coletas de dados). }\end{array}$ & $\begin{array}{l}\text { visão global sobre os métodos de coleta de } \\
\text { dados dos usuário, focando nos estudos dos } \\
\text { usuários. }\end{array}$ \\
\hline 18 & $\begin{array}{l}\text { Thwaites e Simkins, } 2009 \text { [2007] - livro: } \\
\text { Experiential Landscape: an approach to } \\
\text { people, place and space. }\end{array}$ & $\begin{array}{l}\text { técnicas de coleta de dados com abordagem nas } \\
\text { pessoas, lugares e espaços. Destaca-se aqui o } \\
\text { mapeamento de experiência. }\end{array}$ \\
\hline 19 & $\begin{array}{l}\text { Vasconcelos, Villarouco e Soares, } 2010 \text { - } \\
\text { artigo: Contribuição da psicologia } \\
\text { ambiental na análise ergonômica do } \\
\text { ambiente construído. }\end{array}$ & $\begin{array}{l}\text { aplicação, na análise ergonômica do ambiente } \\
\text { construído, da ferramenta constelação de atribu- } \\
\text { tos, advinda da psicologia ambiental. }\end{array}$ \\
\hline
\end{tabular}

Para identificação de quais métodos e técnicas seriam passíveis de coletar dados cognitivos dos usuários de sistemas de wayfinding, foi então realizada uma seleção conforme descrito a seguir. 


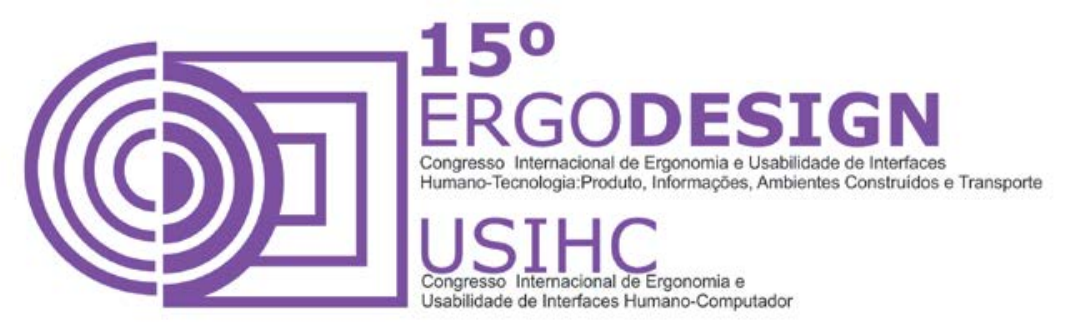

\subsection{Seleção dos Métodos e Técnicas de Coleta de Dados}

A seleção dos métodos e técnicas contou com uma busca por palavras-chave (identificadas na literatura contemplando aspectos de cognição e percepção espacial). Foram escolhidas 13 palavras-chave, considerando algumas variações semânticas, e seus correspondentes na língua inglesa, conforme apresentado no quadro 3.

Quadro 3 - Palavras-chave utilizadas para seleção dos métodos e técnicas

\begin{tabular}{|l|l|}
\hline Palavra-chave & Correspondente em inglês \\
\hline Cognição / Cognitivo & Cognition / Cognitive \\
\hline Espaço/Espacial & Space / Spatial \\
\hline Percepção/Perceptivo/Percebido & Perception / Perceptual / Perceived \\
\hline Comportamento/Comportamental & Behavior / Behavioral \\
\hline Representação/Desenho/Esquema & Representation/Schemata \\
\hline Mapa/Mapeamento & Map/Mapping \\
\hline Localização & Location \\
\hline Mapa mental & Mental map \\
\hline Usuário & User \\
\hline Hospital/Hospitalar/Saúde & Hospital /Healthcare/ Health/ Healthy \\
\hline Ambiente & Environment / Environmental \\
\hline Ação (no espaço) & Action \\
\hline Navegação & Navigation \\
\hline
\end{tabular}

Após a busca através das palavras-chave em cada métodoe técnica anteriormente levantado, foi realizada análise qualitativa através de leitura interpretativa (Gil, 2010), para confirmar se as palavras-chave encontradas realmente estavam relacionadas ao contexto pretendido. Deste modo, foi possível filtrar os 61 métodos e técnicas levantados para coleta de dados em Design Centrado no Usuário, e sintetizá-los em 25 métodos e técnicas com potencial para coleta de dados cognitivos dos usuários, já nas fases iniciais dos projetos de wayfinding de ambientes como os hospitais.

A seleção indicou maior incidência de algumas palavras-chave nos textos dos autores, com destaque à palavra "ambiente" a qual apareceu 17 vezes nos métodos e técnicas. Em seguida os conjuntos de "Comportamento/comportamental", "Representação/desenho/esquema"e cognição/cognitivo/Cognition" apareceram em 13, 12 e 11 descrições consecutivamente. As menores incidências foram das palavras "localização" e "navegação" citadas 3 vezes e, do conjunto "hospital/hospitalar/saúde" o qual foi descrito em apenas 2 métodos e técnicas respectivamente. A palavra-chave "usuário/user" esteve presente em todas descrições, uma vez que, como já mencionado, ela deveria estar explícita nas descrições de todos os métodos e técnicas por se tratar de coleta de dados dos usuários. Vale ressaltar que, a alta ou baixa incidência das palavras-chave nos métodos e técnicas não estão 


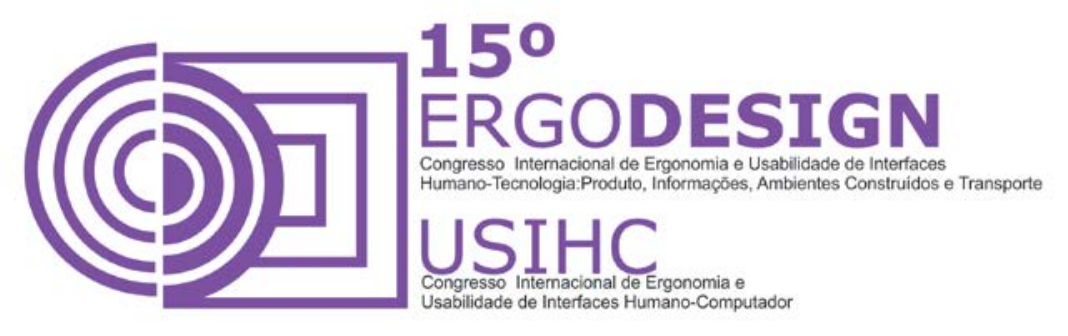

necessariamente ligadas à prioridade dos métodos e técnicas na aplicação em sistemas de wayfinding, já que as incidências verificadas partiram das descrições dos autores selecionados. A escolha do conjunto de técnicas métodos e técnicas pautou-se também na análise desta pesquisadora. Os 25 métodos e técnicas selecionados são descritos no quadro 4.

\section{Quadro 4 - Os 25 métodos e técnicas selecionados}

\begin{tabular}{|c|c|}
\hline Análise Contextual & \multirow{2}{*}{$\begin{array}{l}\text { Percurso cognitivo / Cognitive walk- } \\
\text { through/ Percurso coletivo /Pluralistic } \\
\text { Walkthroughs }\end{array}$} \\
\hline \multirow{2}{*}{$\begin{array}{l}\text { Análise da Tarefa / Investigação } \\
\text { Contextual da Tarefa/ Task analysis / } \\
\text { Contextual Task Inquiry }\end{array}$} & \\
\hline & Personas \\
\hline Análise do Incidente Crítico & Poema dos Desejos / Wish Poem \\
\hline Braindrawing / Desenho de ideias & \multirow{2}{*}{$\begin{array}{l}\text { Protocolo verbal/Verbalização / } \\
\text { Think Aloud }\end{array}$} \\
\hline \multirow{2}{*}{$\begin{array}{l}\text { Card Sorting / } \\
\text { Ordenação por cartões }\end{array}$} & \\
\hline & Rastreamento Ótico/ Eye-tracking \\
\hline \multirow{2}{*}{$\begin{array}{l}\text { Cenários/ Scenarios of use / } \\
\text { Cenário escrito }\end{array}$} & Sense Making \\
\hline & Sondas Culturais / Cultural Probes \\
\hline Constelação de Atributos & \multirow{3}{*}{$\begin{array}{l}\text { Teste de Usabilidade } \\
\text { Entrevista / User Requirements } \\
\text { Interwiew }\end{array}$} \\
\hline Diários / Diary keeping & \\
\hline Diferencial Semântico & \\
\hline Esboço Cartográfico & \multirow{2}{*}{$\begin{array}{l}\text { Observação (não participante, partici- } \\
\text { pante)/Field study /User observa- } \\
\text { tion/Etnografia aplicada /Applied } \\
\text { ethnography }\end{array}$} \\
\hline $\begin{array}{l}\text { Estruturação do Espaço/ } \\
\text { Esquemas de imagem }\end{array}$ & \\
\hline Mapa Mental & Questionário \\
\hline $\begin{array}{l}\text { Mapeamento de experiência / } \\
\text { Mapping Experience }\end{array}$ & Workshops \\
\hline
\end{tabular}

A seleção desses 25 métodos e técnicas passíveis de utilização na fase inicial do processo de design de sistemas de wayfinding para ambientes hospitalares, foi realizada com base nas descrições dos autores que as citaram. Neste artigo não será possível a descrição de cada um dos métodos e técnicas selecionados, espera-se que a citação de cada um sirva como ponto de partida para outras consultas. Apresenta-se assim, um panorama de ferramentas (métodos e técnicas) disponíveis para a conformação de um processo de design de sistemas de wayfinding, centrado no usuário.

Vale ressaltar que o métodos e técnicas foram selecionados de acordo com os critérios adotados, ou seja, aplicáveis à fase inicial dos processos, capazes de coletar dados relativos à cognição/percepção espacial em sistemas de wayfinding de ambientes hospitalares. No entanto, essa aplicação não se esgota aqui, já que, conforme descrito anteriormente, esses métodos e técnicas advêm de diversas áreas de estudo e, com as devidas adaptações, podem ser e são também aplicáveis a diversas situações projetuais que envolvam a cognição/percepção do usuário. 


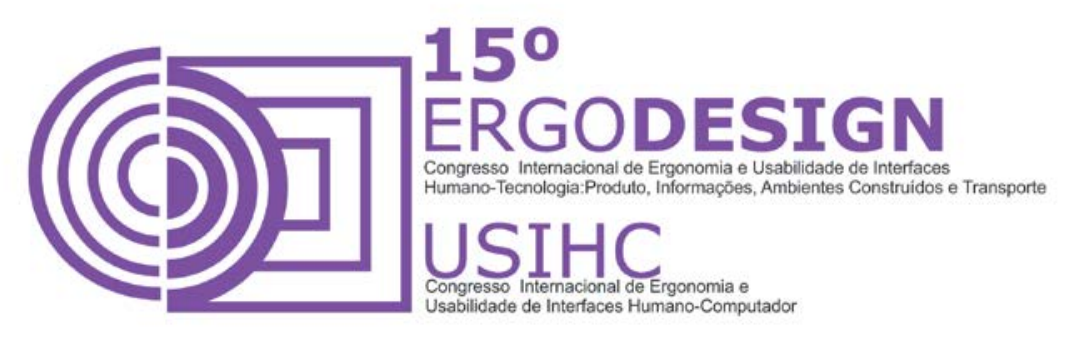

\section{CONSIDERAÇÕES}

Cabe ressaltar que os métodos e técnicas levantados inicialmente e não selecionadas posteriormente, não são, necessariamente, inadequados aos sistemas de wayfinding, apenas não foram considerados passíveis de captação de dados relativos à cognição e percepção espacial. Deste modo, tais métodos e técnicas não foram considerados úteis para utilização no início do processo de design, já que entender como as pessoas percebem e agem no ambiente tende a auxiliar na definição dos artefatos visuais que serão utilizados em sistemas de wayfinding. Tanto no levantamento, quanto na seleção, foram encontrados métodos e técnicas que inicialmente contemplariam os critérios estabelecidos, no entanto, a partir da leitura analítica e interpretativa, esses foram descartados por não possuírem intenções e possibilidade de adaptações para sistemas de wayfinding. Acrescenta-se que essa aplicação não se esgota aqui uma vez que, conforme descrito anteriormente, esses métodos e técnicas advém de diversas áreas de estudo e, com as devidas adaptações podem ser também aplicáveis à diversas situações projetuais que envolvam a cognição/percepção do usuário.

A busca de métodos e técnicas de coleta de dados específicos para sistemas de wayfinding em hospitais não foi profícua. Foram encontradas apenas recomendações sobre aspectos relevantes a serem levantados no ambiente hospitalar, e menção à importância de coletar dados dos usuários ( MOURSHED e ZHAO, 2012; MOLLERUP, 2005). No entanto, a literatura de Design Centrado no Usuário/Pessoa (incluindo Interação Humano-Computador e Ergonomia) assim como dos estudos sobre os usuários, advindos da Ciência da Informação, possibilitaram uma ampla gama de possibilidades de métodos e técnicas capazes de serem utilizadas em sistemas de wayfinding.

Mesmo com o resultado do levantamento e posterior seleção, não se pode afirmar que os profissionais de sistemas de wayfinding conheçam, concordem, utilizem ou queiram utilizar tais métodos e técnicas. Deste modo, validação dos dados levantados junto ao público de interesse foi realizada, porém os procedimentos e resultados não são pertinentes a esse trabalho e serão descritos em outro artigo. Este trabalho trouxe informações que podem subsidiar profissionais no incremento de seus projetos de wayfinding. Desta forma, espera-se que essas informações sirvam de contribuições para as diferentes áreas como ergonomia, design e arquitetura e estimulem profissionais a inserirem os usuários já nas fases iniciais do processo de design para sistemas de wayfinding, aumentando assim o potencial de sucesso em tais sistemas.

\section{REFERÊNCIAS BIBLIOGRÁFICAS}

ANSI- American National Standards Institute. 2006. ANSI Z535.1-(R2011). Disponível em: $<$ http://www.ansi.org/> Acesso em jan 2013

ARTHUR, P. e PASSINI, R. Wayfinding-People, Signs, and Architecture. New York: McGraw-Hill, [1992], 2002. 


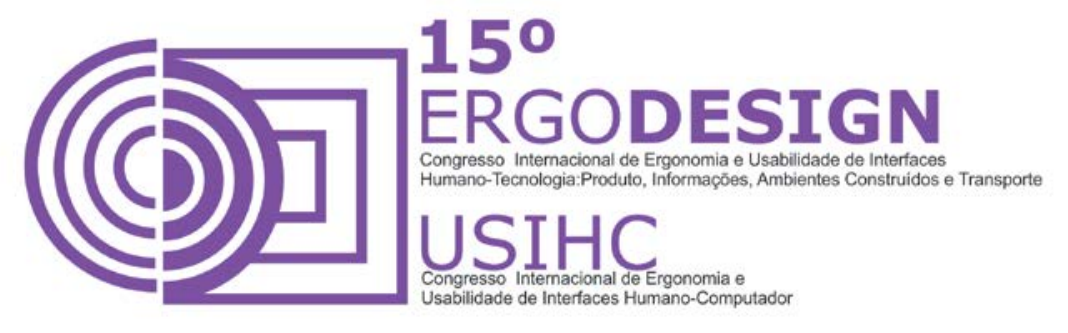

BERGER, C. Wayfinding: Designing and Implementing Graphic Navigational Systems. Rotovision Switzerland. 2009.

DEWAR, R. Design and evaluation of public information symbols. Visual Information for everyday use: design and research perspectives. London: Taylor \& Francis, 1999.

DOWNS, R. N. e STEA, D. Maps in Minds: Reflections on Cognitive Mapping. NewYork: Harper \& Row. 1977.

GIBSON, D. The Wayfinding Handbook: Information Design for Public Places. Princeton. Architectural Press, 2009.

GIL, A. C. Como elaborar projetos de pesquisa. 5ª ed. São Paulo: Atlas [1991] 2010.

GOLLEDGE, R. G. Wayfinding Behavior: Cognitive Mapping and Other Spacial Process. Johns Hopkins University Press, EUA, 1999.

ISO 9241-210, Ergonomics of human-system interaction - Part 210: Human-centred design for interactive systems, 2010.

ISO WD 9241-230.Human-centred design and evaluation methods - Part 230: Usability methods supporting human centred design. 2009

ISTOMIN, K. V.; DWYER, M. J. A. Critical Discussion of Anthropological Theories of Human Spatial Orientation with Reference to Reindeer Herders of Northeastern Europe and Western Siberia. In: Current Anthropology, v.50, n.01, NY, USA. 2009.

LYNCH, K. A Imagem da Cidade. $1^{a}$ ed. brasileira, $2^{a}$ reimpressão. São Paulo: Martins Fontes. $\left.\right|^{a}$ ed. Cambridge: The M.I.T. Press [1960] 1999.

MAGUIRE, M. Methods to support human-centred design. International Journal of Human-Computer Studies. v. 55, Issue 4, p. 587-634, October, 2001.

MIJKSENAAR, P. Maps as public graphics: about science and craft, curiosity and passion. In Visual information for everyday use: design and research perspectives. London: Taylor \& Francis, 1999.

MILLER, C., e LEWIS, D. Wayfinding in complex healthcare environments. In: Information Design Journal, 9(2\&3), 2000.

MOLLERUP, P. Wayshowing: A Guide to Environmental Signage Principles and Practices. Baden, Suíça: Lars Muller. 2005.

MOLLERUP, P. Wayshowing in Hospital. In: Australasian Medical Journal, 1, 10, 2009.

MOURSHED, M. e ZHAO, Y. Healthcare providers' perception of design factors related to physical environments in hospitals. In: Journal of Environmental Psychology, 2012. 


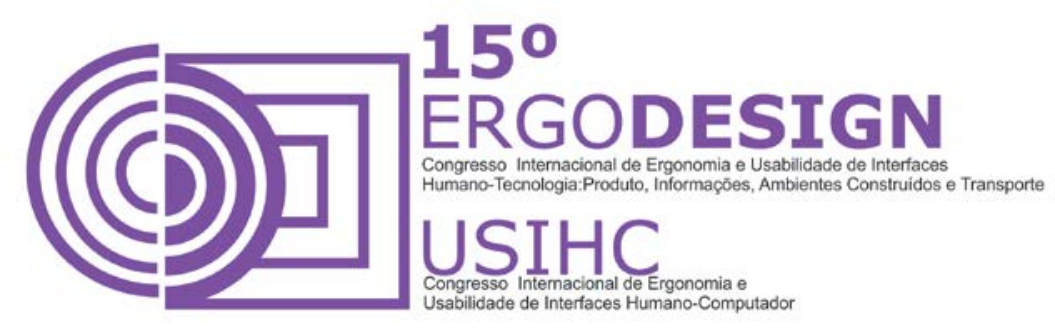

O'NEILL, M. Theory and research in design of 'you are here' maps. In: ZWAGA, H.; BOERSEMA, T.; HOONHOUT, H. Visual information for everyday use: design and research perspectives. London: Taylor \& Francis, 1999.

PADOVANI, S.; MOURA, D. Navegação em Hipermídia: Uma abordagem centrada no usuário. Rio de Janeiro. Ed. Ciência Moderna. 2008.

PADOVANI, S. et al. Trilhando o caminho de volta: um estudo da adaptação de métodos de design centrado no usuário para sistemas de informação digitais visando aplicação a mídia impressa. p.5938-5951, 2012. Disponível na internet por http em: <http://www.peddesign2012.ufma.br/anais/Anais/anais10PeD2012.part2.pdf > Acesso em: abril 2013

RAUBAL, M., et al. Structuring Space with Image Schemata: Wayfinding in Airports as a Case Study. 1997. In: Proceedings of the International Conference on Spatial Information Theory. Disponível na internet por http em: <ftp://ftp.geoinfo.tuwien.ac.at/raubal/cosit97_raubal.pdf> Acesso em: mar. 2011

SIEGEL, A. W.; WHITE, S. H. The development of spatial representations of large scale environments. In Reese, H. W. (ed.), Advances in Child Development and Behavior, v. 10: 9-55. Academic Press, London. 1975.

\section{AGRADECIMENTOS}

À Coordenação de Aperfeiçoamento de Pessoal de Nível Superior - CAPES pelo incentivo à pesquisa através da bolsa de auxílio financeiro para o mestrado. 